\title{
Effect of calcium channel blockers on incidence of diabetes: a meta-analysis
}

This article was published in the following Dove Press journal:

Diabetes, Metabolic Syndrome and Obesity:Targets and Therapy

25 July 2013

Number of times this article has been viewed

\author{
Hiroshi Noto ${ }^{1,2}$ \\ Atsushi Goto ${ }^{2}$ \\ Tetsuro Tsujimoto' \\ Mitsuhiko Noda ${ }^{1,2}$ \\ 'Department of Diabetes and \\ Metabolic Medicine, Center \\ Hospital, National Center for Global \\ Health and Medicine, Tokyo, Japan; \\ ${ }^{2}$ Department of Diabetes Research, \\ Diabetes Research Center, Research \\ Institute, National Center for Global \\ Health and Medicine, Tokyo, Japan
}

\begin{abstract}
Aims: Insulin resistance and the progressive loss of $\beta$-cell function are components of the fundamental pathophysiology of type II diabetes. A recent experimental study suggested that calcium channel blockers (CCBs) might inhibit $\beta$-cell apoptosis, enhance $\beta$-cell function, and prevent diabetes. The present meta-analysis examined the clinical effect of CCBs on the incidence of diabetes.
\end{abstract}

Methods: MEDLINE, EMBASE, ISI Web of Science, the Cochrane Library, and ClinicalTrials. gov were each searched for relevant articles published up to March 11, 2013. Randomized controlled trials (RCTs) with a follow-up period of at least 1-year were included. Identified articles were systematically reviewed, and those with pertinent data were selected for inclusion in a meta-analysis.

Results: We included ten RCTs in a meta-analysis. Of the 108,118 people with hypertension and no pre-existing diabetes, 7,073 (6.5\%) cases of type II diabetes were reported. CCBs were associated with a higher incidence of diabetes than angiotensin-converting enzyme inhibitors (ACEIs) or angiotensin-receptor blockers (ARBs; pooled risk ratios [95\% confidence intervals]: 1.23 [1.01-1.51] and 1.27 [1.14-1.42], respectively) and a lower incidence compared with $\beta$ blockers or diuretics $(0.83$ [0.73-0.94] and 0.82 [0.69-0.98], respectively). The overall risk of diabetes among subjects taking CCBs was not significant (0.99 [0.85-1.15]).

Conclusion: The use of CCBs was not significantly associated with incident diabetes compared to other antihypertensive agents: the association with diabetes was lowest for ACEIs and ARBs, followed by CCBs, $\beta$ blockers, and diuretics. Although CCBs can be safely used in hypertensive patients, it would be premature to advocate CCBs for the prevention or treatment of diabetes.

Keywords: diabetes, calcium channel blockers, hypertension, meta-analysis

\section{Introduction}

A growing body of evidence has suggested that the effects of different classes of antihypertensive medications on the incidence of diabetes vary, with the lowest association reported for angiotensin-converting enzyme inhibitors (ACEIs) and angiotensin-receptor blockers (ARBs) followed by calcium channel blockers (CCBs), $\beta$ blockers, and diuretics. ${ }^{1}$ The progressive loss of pancreatic $\beta$-cell mass/function is a key component in the pathogenesis of both type I and type II diabetes and also underlies insulin resistance in type II diabetes. A recent study using human islet cells and murine models for type I and type II diabetes demonstrated that verapamil, a CCB, might inhibit the expression of the proapoptotic $\beta$-cell thioredoxin-interacting protein (TXNIP) in INS-1 cells and human islets, thereby enhancing $\beta$-cell survival and function and preventing diabetes in BTBR ob/ob mice. ${ }^{2}$
Correspondence: Hiroshi Noto Department of Diabetes and Metabolic Medicine, Center Hospital, National Center for Global Health and Medicine, I-2 I-I Toyama, Shinjuku,

Tokyo 162-8655, Japan

Tel +8I 332027181

Fax +81332071038

Email noto-tky@umin.net 
In light of the worldwide epidemics of diabetes and hypertension, explorations of the effect of antihypertensive drugs on the incidence of diabetes are of clinical importance. Moreover, they are crucial in the areas of public health, since a modest increase in the risk of diabetes translates into a substantial social burden. These circumstances prompted us to investigate, with greater precision, the effects of CCBs on diabetes prevention by scrutinizing pertinent up-to-date original reports and combining their data in an attempt to obtain meaningful clues for an evaluation of the potential benefits of CCBs.

\section{Methods}

Searches of MEDLINE, EMBASE, ISI Web of Science, the Cochrane Library, and ClinicalTrials.gov from their inception until March 11, 2013, were performed. Studies evaluating the incidence of diabetes among subjects taking CCBs, compared to those taking other antihypertensive medications, were identified using a combination of the following keywords: "calcium channel blocker" and "diabetes mellitus". The reference lists of the pertinent articles were also inspected. We assessed all the identified studies with regard to the effects of CCBs on the incidence of diabetes based on original data analyses to determine their eligibility for inclusion in a qualitative analysis. The inclusion criteria for the metaanalysis were as follows: a published full-text report, the use of a randomized controlled trial (RCT) with a follow-up period of at least 1-year, and the reporting of event numbers. To ascertain the validity of the eligible studies, the quality of each report was appraised in reference to the CONSORT statement ${ }^{3}$ and the QUOROM statement. ${ }^{4}$ We then reviewed each full-text report to determine its eligibility and extracted and tabulated all the relevant data independently. The extracted data included the characteristics of the subjects (including age, sex, and region), published year, follow-up period, outcomes, and the methods used for risk estimation. Any disagreement was resolved by a consensus among the investigators. If more than one study was published for the same subjects with identical endpoints, the report containing the most comprehensive information on the population was included to avoid overlapping populations.

In the meta-analysis, the relative risks with CCBs compared with each comparator were combined and the pooled risk ratio $(\mathrm{RR})$ with the $95 \%$ confidence interval $(\mathrm{CI})$ was calculated using the Mantel-Haenszel random-effects model. Heterogeneity among the studies was evaluated using the $\mathrm{I}^{2}$ statistics. A subgroup analysis and a meta-regression analysis ${ }^{5}$ were also conducted, as appropriate. The equality of the risk ratios between subgroups was assessed using the z-statistic test. RevMan (version 5.2, The Nordic Cochrane Centre, The Cochrane Collaboration, Copenhagen, Denmark) was used for these calculations. All the procedures were performed in accordance with the PRISMA statement. ${ }^{6}$

\section{Results}

A total of 1,146 articles were identified during our search; of these, $12^{7-18}$ were assessed with respect to their eligibility for inclusion in our review, which was aimed at determining the influence of $\mathrm{CCBs}$ on the incidence of diabetes. Out of these 12 articles, one study ${ }^{10}$ in which CCBs were compared with a placebo and another ${ }^{18}$ in which there was no event excluded; consequently, ten RCTs were included in the meta-analysis (Figure 1). The selected articles were moderately heterogeneous in terms of the population demographics. Most of the included studies were conducted in the United States and European countries, and their follow-up durations (range: 2.7-5.5 years) were sufficiently long for the outcomes to occur.

Of the total of 108,118 people with hypertension and no pre-existing diabetes, 7,073 (6.5\%) cases of type II diabetes were reported. The RRs against each comparator and the overall RR are depicted in Figure 2. CCBs were associated with a higher incidence of diabetes, compared to ACEIs $(\mathrm{n}=3)^{7,8,14}$ or ARBs $(\mathrm{n}=2$; pooled RR [95\% CI], 1.23 [1.01-1.51] and $\mathrm{I}^{2}=27 \%, 1.27[1.14-1.42]$ and $\mathrm{I}^{2}=2 \%$, respectively), ${ }^{15,16}$ and a lower incidence compared with $\beta$ blockers $(n=5)^{7,9,12-14}$ or diuretics $(n=3$; pooled $R R, 0.83$ [0.73-0.94] and $\mathrm{I}^{2}=61 \%, 0.82[0.69-0.98]$ and $\mathrm{I}^{2}=19 \%$, respectively). ${ }^{8,11,17}$ As described in Table 1, the RRs against newer modalities (ACEIs and ARBs) were significantly higher than those against traditional agents ( $\beta$ blockers and diuretics). Only two reports investigated the risk associated with non-dihydropyridines against $\beta$ blockers, ${ }^{12,13}$ which was not statistically different from the risk associated with dihydropyridines against $\beta$ blockers $(n=3) .^{7,9,14}$ The overall risk of diabetes among those with $\mathrm{CCBs}$ was nonsignificant (pooled RR, 0.99 [0.85-1.15] and $\mathrm{I}^{2}=87 \%$ ). The heterogeneity among the reports in each medication category and for the overall analysis was generally high. The risk was consistent despite differences in age, the proportion of men, the risk of cardiovascular disease, and blood pressure between the randomized groups (Table 1).

\section{Discussion}

Our meta-analyses of worldwide RCTs suggested that the metabolic effect of CCBs was neutral, compared to other 


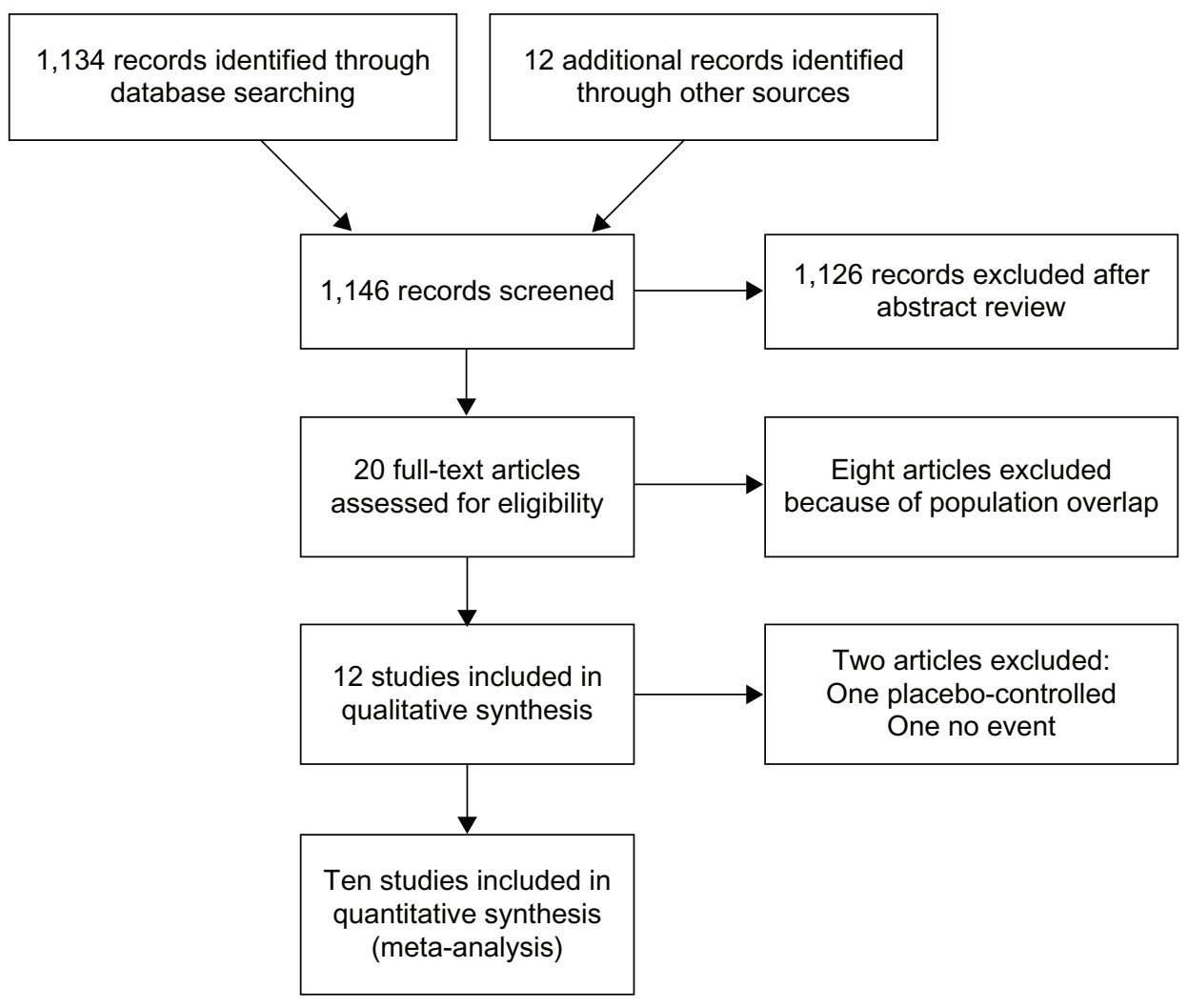

Figure I Flow diagram of study selection.

antihypertensive drugs, which did not support the notion that CCBs might prevent diabetes ${ }^{2}$ or refute the current guidelines for compelling indications. ${ }^{19,20}$ For those with overt diabetes and hypertension, ACEIs and ARBs are generally preferred in light of the lower risk of diabetes progression, as suggested by our analysis and the nephroprotective effects of these agents.

The strength of our present study is that the analysis was solely based on long-term, large-scale RCTs originating from multiple nations and was thus more comprehensive than previous articles. ${ }^{1,21}$ The included data were good quality, apparently had sufficient power to detect differences in the risk of the outcome, and were biologically plausible. The temporal sequence of the events was appropriate. The outcome ascertainment tools were valid, and there were few, if any, missed confounders. The heterogeneity of the results within each comparator group was low except for $\beta$ blockers: this low heterogeneity suggests that each of the results was consistent and that most of the variation was attributable to chance alone. The large $\mathrm{I}^{2}$ values in some analyses indicated that the range of the plausible risk estimates was wide, generally because of the diversity of the study design, population backgrounds, and ethnicities. The heterogeneity of the overall analysis was quite high, probably because of the variety of drug classes that were confounded by indication. The extent of blood pressure change was possibly another source of heterogeneity, but this hypothesis cannot be statistically tested in light of the scarcity of data. A subgroup analysis and a meta-regression, which suggested a consistent risk regardless of the CCB subclass, age, sex, cardiovascular risk, or difference in achieved blood pressure, support the safety and the clinical generalizability of CCBs.

Verapamil was recently reported to inhibit TXNIP expression in INS-1 cells and human islets, and orally administered verapamil reduced TXNIP expression and $\beta$-cell apoptosis, enhanced endogenous insulin levels, and rescued mice from streptozotocin-induced diabetes. ${ }^{2}$ Verapamil also reportedly promoted $\beta$-cell survival and improved glucose homeostasis and insulin sensitivity in BTBR ob/ob mice. ${ }^{2}$ Despite these facts, our study did not find a metabolic benefit. Thus, the magnitude of the protective effect may be too small to have an effect on type II diabetes in clinical settings, and trials in patients with type I diabetes may provide some insights. Further studies to scrutinize the effect of CCBs on glycemic control in diabetic patients are also pending.

Although the quality of the included studies was high, our analysis should be interpreted in the context of the following limitations. The number of available studies for each 


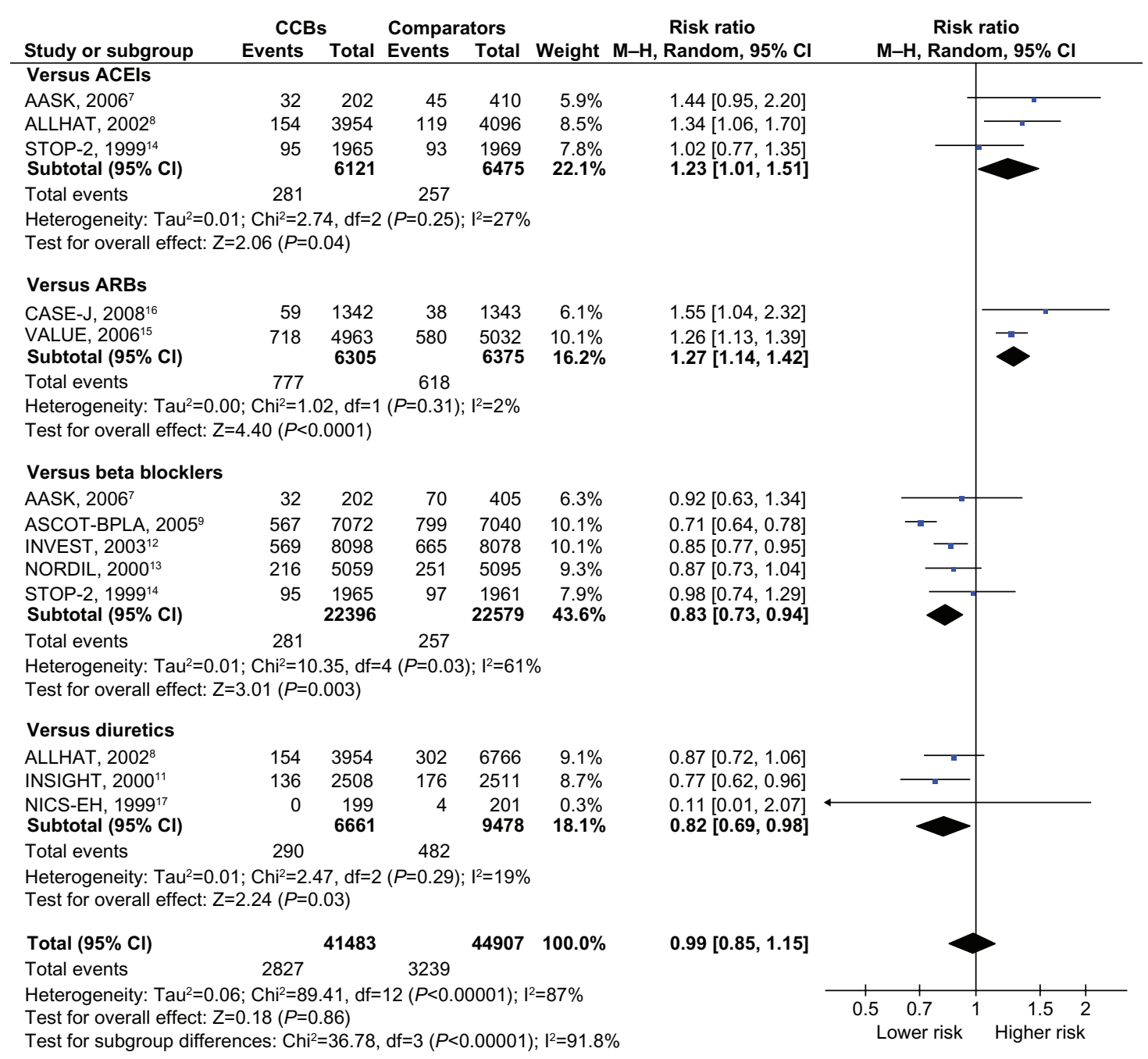

Figure 2 Risk ratios for diabetes associated with $\mathrm{CCBs}$, compared to other antihypertensive drugs.

Notes: Boxes, estimated risk ratios (RRs); bars, $95 \% \mathrm{Cl}$. Diamonds, fixed-effects model RRs; width of diamonds, pooled Cls. The size of each box is proportional to the weight of each study in the meta-analysis.

Abbreviations: AASK, African American Study of Kidney diseases and hypertension; ACEl, Angiotensin-Converting Enzyme Inhibitor; ALLHAT, Antihypertensive and Lipid Lowering to prevent Heart Attack Trial; ARB, angiotensin-receptor blocker; ASCOT-BPLA, Anglo-Scandinavian Cardiac Outcomes Trial-Blood Pressure Lowering Arm; CASE-J, Candesartan Antihypertensive Survival Evaluation in Japan; CCB, calcium channel blocker; INSIGHT, International Nifedipine; GITS Study, Intervention as a Goal in Hypertension Treatment Study; INVEST, International Verapamil-Trandolapril Study; NICS-EH, National Intervention Cooperative Study in Elderly Hypertensives; NORDIL, Nordic Diltiazem study; M-H, Mantel-Haenszel; STOP-2, Second Swedish Trial in Older Patients with hypertension-2; VALUE, Valsartan Antihypertensive Long-term Use Evaluation.

medication subclass was relatively small and the studies were moderately heterogeneous, especially for $\beta$ blockers. Thus, a publication bias may exist, although we cannot assess this hypothesis. Regarding the external validity of the results, it is also important to realize that the participants of the studies may not represent general populations. The incident of diabetes was not the primary endpoint in the majority of the included trials and thus diagnostic ascertainment may not have been valid.

Although CCBs might inhibit proapoptotic $\beta$-cell TXNIP expression, thereby enhancing $\beta$-cell survival and function, we found that the use of CCBs was not significantly associated with incident diabetes compared to other antihypertensive agents. Further studies on the complex interactions between CCBs and TXNIP are warranted before CCBs can be advocated as a measure for diabetes prevention or treatment.

\section{Acknowledgments}

All the authors had full access to all the data in the study and take responsibility for the integrity of the data and the accuracy of the data analysis.

Conceived and designed the experiments: HN, MN. Performed the experiments: HN, AG, TT. Analyzed the data: HN, AG, TT. Contributed reagents/materials/analysis tools: HN, AG, TT. Wrote the paper: HN. Reviewed/edited the manuscript: AG, TT, MN.

This study was supported by a Health Sciences Research Grant (Comprehensive Research on Diabetes/Cardiovascular and Life-Style Related Diseases H22-019) from the Ministry of Health, Labor and Welfare of Japan. The funders had no role in the study design, data collection and analysis, decision to publish, or the preparation of the manuscript. 


\section{Disclosure}

The authors report no conflicts of interest in this work.

\section{References}

1. Elliott WJ, Meyer PM. Incident diabetes in clinical trials of antihypertensive drugs: a network meta-analysis. Lancet. 2007;369(9557): 201-207.

2. Xu G, Chen J, Jing G, Shalev A. Preventing \#beta-cell loss and diabetes with calcium channel blockers. Diabetes. 2012;61(4):848-856.

3. Schulz KF, Altman DG, Moher D; CONSORT Group. CONSORT 2010 statement: updated guidelines for reporting parallel group randomised trials. Int J Surg. 2011;9(8):672-677.

4. Moher D, Cook DJ, Eastwood S, Olkin I, Rennie D, Stroup DF. Improving the quality of reports of meta-analyses of randomised controlled trials: the QUOROM Statement. Onkologie. 2000;23(6):597-602.

5. Thompson SG, Higgins JP. How should meta-regression analyses be undertaken and interpreted? Stat Med. 2002;21(11):1559-1573.

6. Liberati A, Altman DG, Tetzlaff J, et al. The PRISMA statement for reporting systematic reviews and meta-analyses of studies that evaluate health care interventions: explanation and elaboration. Ann Intern Med. 2009;151(4):W65-W94.

7. Thornley-Brown D, Wang X, Wright JT, et al. Differing effects of antihypertensive drugs on the incidence of diabetes mellitus among patients with hypertensive kidney disease. Arch Intern Med. 2006;166(7):797-805.

8. ALLHAT Officers and Coordinators for the ALLHAT Collaborative Research Group. The Antihypertensive and Lipid-Lowering Treatment to Prevent Heart Attack Trial. Major outcomes in high-risk hypertensive patients randomized to angiotensin-converting enzyme inhibitor or calcium channel blocker vs diuretic: The Antihypertensive and LipidLowering Treatment to Prevent Heart Attack Trial (ALLHAT). JAMA. 2002;288(23):2981-2997.

9. Dahlöf B, Sever PS, Poulter NR, et al; ASCOT Investigators. Prevention of cardiovascular events with an antihypertensive regimen of amlodipine adding perindopril as required versus atenolol adding bendroflumethiazide as required, in the Anglo-Scandinavian Cardiac Outcomes Trial-Blood Pressure Lowering Arm (ASCOT-BPLA): a multicentre randomised controlled trial. Lancet. 2005;366(9489):895-906.

10. Liu L, Zhang Y, Liu G, et al; FEVER Study Group. The Felodipine Event Reduction (FEVER) Study: a randomized long-term placebo-controlled trial in Chinese hypertensive patients. $J$ Hypertens. 2005;23(12): 2157-2172.

11. Brown MJ, Palmer CR, Castaigne A, et al. Morbidity and mortality in patients randomised to double-blind treatment with a long-acting calcium-channel blocker or diuretic in the International Nifedipine GITS study: Intervention as a Goal in Hypertension Treatment (INSIGHT). Lancet. 2000;356(9227):366-372.
12. Pepine CJ, Handberg EM, Cooper-DeHoff RM, et al; INVEST Investigators. A calcium antagonist vs a non-calcium antagonist hypertension treatment strategy for patients with coronary artery disease. The International Verapamil-Trandolapril Study (INVEST): a randomized controlled trial. JAMA. 2003;290(21):2805-2816.

13. Hansson L, Hedner T, Lund-Johansen P, et al. Randomised trial of effects of calcium antagonists compared with diuretics and beta-blockers on cardiovascular morbidity and mortality in hypertension: the Nordic Diltiazem (NORDIL) study. Lancet. 2000;356(9227):359-365.

14. Hansson L, Lindholm LH, Ekbom T, et al. Randomised trial of old and new antihypertensive drugs in elderly patients: cardiovascular mortality and morbidity the Swedish Trial in Old Patients with Hypertension-2 study. Lancet. 1999;354(9192):1751-1756.

15. Kjeldsen SE, Julius S, Mancia G, et al; VALUE Trial Investigators. Effects of valsartan compared to amlodipine on preventing type 2 diabetes in high-risk hypertensive patients: the VALUE trial. J Hypertens. 2006;24(7):1405-1412.

16. Ogihara T, Nakao K, Fukui T, et al; Candesartan Antihypertensive Survival Evaluation in Japan Trial Group. Effects of candesartan compared with amlodipine in hypertensive patients with high cardiovascular risks: candesartan antihypertensive survival evaluation in Japan trial. Hypertension. 2008;51(2):393-398.

17. Randomized double-blind comparison of a calcium antagonist and a diuretic in elderly hypertensives. National Intervention Cooperative Study in Elderly Hypertensives Study Group. Hypertension. 1999;34(5): 1129-1133.

18. Kaneshiro Y, Ichihara A, Sakoda M, Kurauchi-Mito A, Kinouchi K, Itoh $\mathrm{H}$. Add-on benefits of amlodipine and thiazide in nondiabetic chronic kidney disease stage 1/2 patients treated with valsartan. Kidney Blood Press Res. 2009;32(1):51-58.

19. Mancia G, De Backer G, Dominiczak A, et al; ESH-ESC Task Force on the Management of Arterial Hypertension. 2007 ESH-ESC Practice Guidelines for the Management of Arterial Hypertension: ESH-ESC Task Force on the Management of Arterial Hypertension. J Hypertens. 2007;25(9):1751-1762.

20. Chobanian AV, Bakris GL, Black HR, et al; National High Blood Pressure Education Program Coordinating Committee. The Seventh Report of the Joint National Committee on Prevention, Detection, Evaluation, and Treatment of High Blood Pressure: the JNC 7 report. JAMA. 2003;289(19):2560-2572.

21. Kuti EL, Baker WL, White CM. The development of new-onset type 2 diabetes associated with choosing a calcium channel blocker compared to a diuretic or beta-blocker. Curr Med Res Opin. 2007;23(6): 1239-1244.

Diabetes, Metabolic Syndrome and Obesity: Targets and Therapy

Dovepress

\section{Publish your work in this journal}

Diabetes, Metabolic Syndrome and Obesity: Targets and Therapy is an international, peer-reviewed open-access journal committed to the rapid publication of the latest laboratory and clinical findings in the fields of diabetes, metabolic syndrome and obesity research. Original research, review, case reports, hypothesis formation, expert opinion and commentaries are all considered for publication. The manuscript management system is completely online and includes a very quick and fair peer-review system, which is all easy to use. Visit http://www.dovepress.com/testimonials.php to read real quotes from published authors. 